\title{
Editorial
}

\section{Optimal Control Techniques in Aircraft Guidance and Control}

\author{
Ernesto Staffetti $\left(\mathbb{D},{ }^{1}\right.$ Xiang Li $\mathbb{D},{ }^{2}$ Yoshinori Matsuno $\left(\mathbb{D},{ }^{3}\right.$ and Manuel Soler $\mathbb{D D}^{4}$ \\ ${ }^{1}$ University Rey Juan Carlos, Madrid, Spain \\ ${ }^{2}$ Beijing Institute of Technology, Beijing, China \\ ${ }^{3}$ Japan Aerospace Exploration Agency, Tokyo, Japan \\ ${ }^{4}$ University Carlos III of Madrid, Madrid, Spain \\ Correspondence should be addressed to Ernesto Staffetti; ernesto.staffetti@urjc.es
}

Received 23 May 2019; Accepted 27 June 2019; Published 5 August 2019

Copyright (C) 2019 Ernesto Staffetti et al. This is an open access article distributed under the Creative Commons Attribution License, which permits unrestricted use, distribution, and reproduction in any medium, provided the original work is properly cited.

Optimal control is a branch of mathematics that deals with analytical and numerical methods to design control strategies for dynamical systems in which some measures of performance are optimised.

Applications of optimal control in aeronautical engineering include aircraft guidance and control. Aircraft guidance refers to the determination of the trajectory to steer the aircraft between two different states. Techniques for aircraft guidance are in general open loop. Aircraft control refers to the determination of the control inputs to follow the planned trajectories while maintaining aircraft stability and smooth manoeuvring. In this case, closed-loop control techniques must be employed.

Although optimal control has been applied in aeronautical engineering for several decades, the continuous technological development in this field is resulting in leading to optimal control models of increasing size and complexity. As a consequence, and despite the progress in the development of numerical solution methods, the increasing size and complexity of the problems generates a constant demand for faster and more efficient methods to solve optimal control problem.

Among the most demanding problems from the computational point of view are the stochastic optimal control problems, in which uncertainties are taken into account and the obtained solution must fulfil probabilistic constraints.

The purpose of this special issue is to bring recognition to the contribution of optimal control techniques to aircraft guidance and control and to provide a forum to disseminate the latest research work with the aim of further stimulating interest in this area of great potential.

The special issue includes several high-quality papers written by leading and emerging specialists in the field.

14 submissions have been received. After a rigorous refereeing process, 5 papers have been accepted for publication in this special issue.

A short description of the addressed topics is presented.

In "Efficient Convex Optimization of Reentry Trajectory via the Chebyshev Pseudospectral Method" by C.-M. Yu et al., optimal control techniques based on the Chebyshev pseudospectral method and on a novel sequential convex optimisation scheme have been applied to solve the hypersonic reentry trajectory optimisation problem.

In "Analyzing the Departure Runway Capacity Effects of Integrating Optimized Continuous Climb Operations" by M. V. Díaz et al., optimal control techniques based on the Chebyshev pseudospectral method have been applied to study the effects of the introduction of continuous climb operations on runway capacity.

In "Characterization and Enhancement of Flight Planning Predictability under Wind Uncertainty" by J. GarcíaHeras et al., potential enhancements in flight planning predictability due to the effects of wind uncertainty are analyzed using a robust optimal control methodology. In this work, wind uncertainty is retrieved out of ensemble probabilistic forecasts.

In "Evasion-Pursuit Strategy against Defended Aircraft Based on Differential Game Theory" by Q. Sun et al., an 
optimal guidance law based on differential game theory is derived for the attacker in an active defence scenario, in which the attacker evades from the defender and pursues the target.

In "Cooperative Guidance of Seeker-Less Missiles for Precise Hit" by J. Zhao and F. Xiong, linear-quadratic optimal control and biased proportional navigation guidance are employed to develop a two-stage cooperative guidance law for fire-and-forget attacks for seeker-less missiles.

\section{Conflicts of Interest}

We declare that none of the authors of the editorial have a conflict of interest.

Ernesto Staffetti

Xiang Li

Yoshinori Matsuno

Manuel Soler 


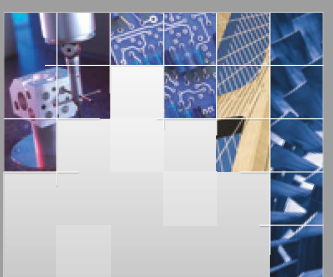

\section{Enfincering}
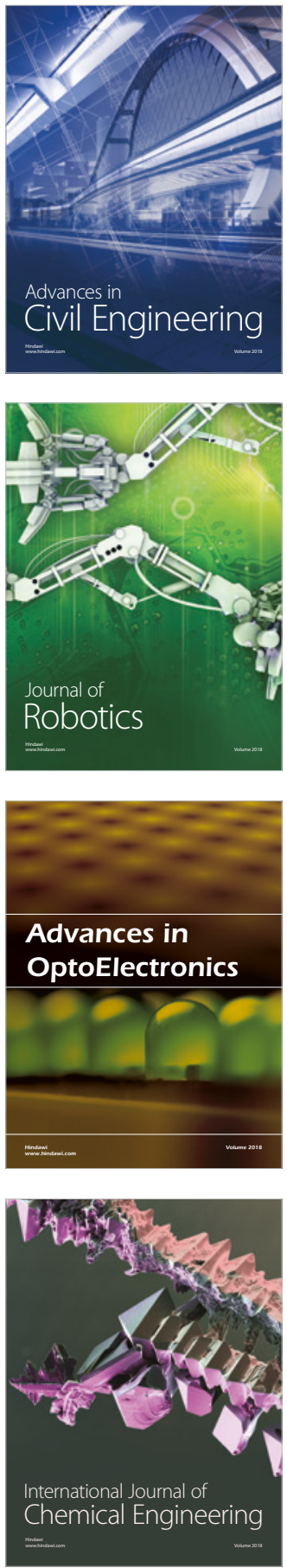

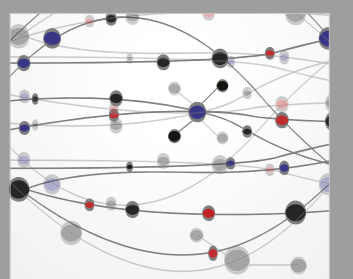

\section{Rotating \\ Machinery}

The Scientific World Journal

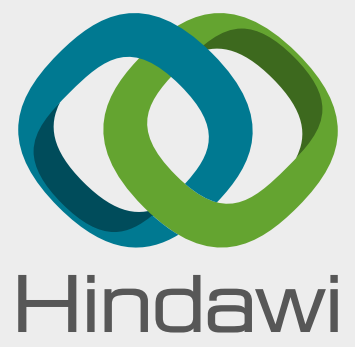

Submit your manuscripts at

www.hindawi.com
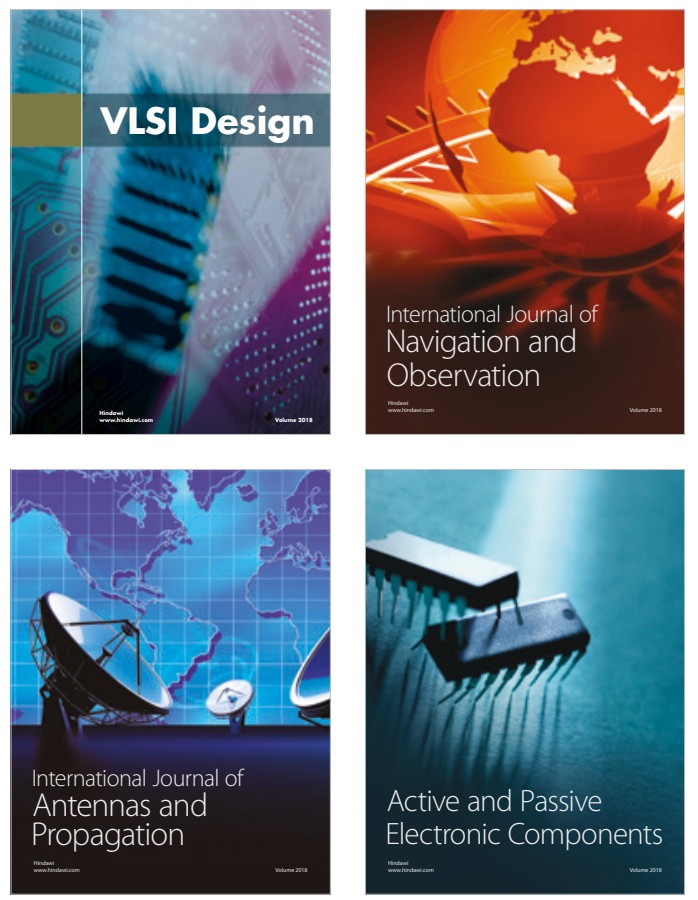
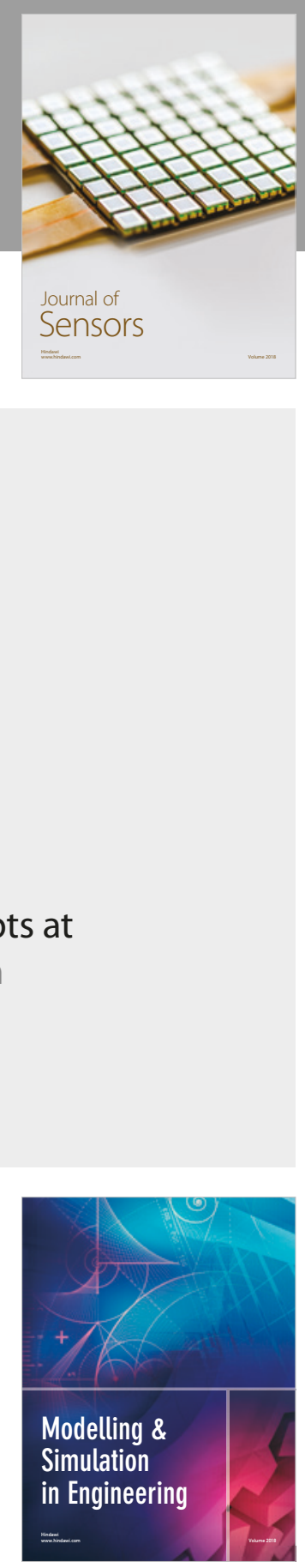

\section{Advances \\ Multimedia}
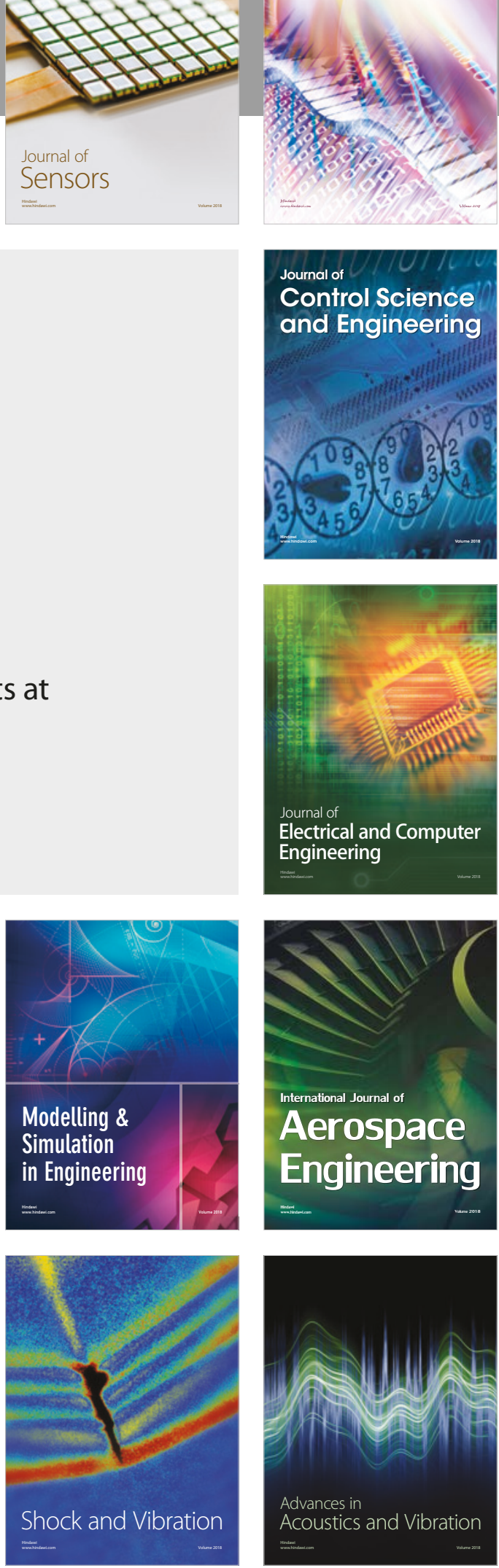